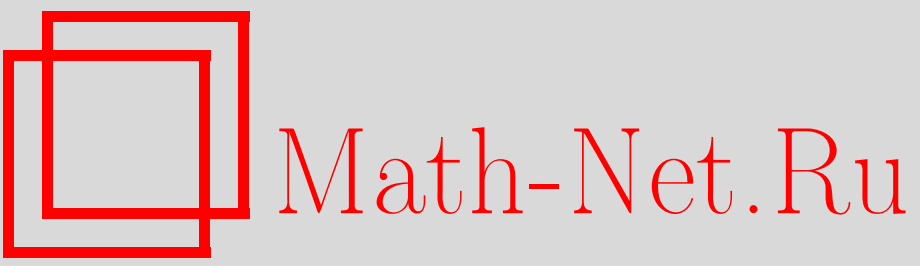

В. Е. Зобов, А. А. Лундин, Моделирование многочастичных когерентностей в ядерных спиновых системах твердых тел посредством взаимодействия с бесконечным радиусом, ТМФ, 2004, том 141, номер 3, 469-484

DOI: https://doi.org/10.4213/tmf135

Использование Общероссийского математического портала Math-Net.Ru подразумевает, что вы прочитали и согласны с пользовательским соглашением

http: //www . mathnet.ru/rus/agreement

Параметры загрузки:

IP : 107.22 .136 .117

26 апреля 2023 г., 13:09:47 


\section{МОДЕЛИРОВАНИЕ МНОГОЧАСТИЧНЫХ КОГЕРЕНТНОСТЕЙ В ЯДЕРНЫХ СПИНОВЫХ СИСТЕМАХ ТВЕРДЫХ ТЕЛ ПОСРЕДСТВОМ ВЗАИМОДЕЙСТВИЯ С БЕСКОНЕЧНЫМ РАДИУСОМ}

На основе точно решаемой модели, известной в статистической механике как модель с бесконечным радиусом взаимодействия (модель Ван-дер-Ваальса), получены выражения для иерархии многоспиновых многоквантовых временны́х корреляционных функций парамагнитной ядерной спиновой системы твердого тела с магнитным дипольдипольным взаимодействием между ядрами. Эти функции наблюдаются экспериментально с помощью методов современной многоквантовой ЯМР-спектроскопии. Явно выписан полный набор ортогональных многоспиновых операторов. Найдена зависимость от времени амплитуд многочастичных временны́х корреляционных функций. Предложенная модель хорошо описывает поведение многочастичных корреляций, по крайней мере для не очень больших номеров гармоник разложения $n$. Результаты теории удовлетворительно согласуются с экспериментальными данными, полученными для гексаметилбензола.

Ключевые слова: многоимпульсный метод, диполь-дипольное взаимодействие, многоспиновая когерентность, временна́я корреляционная функция, врашающаяся система координат.

\section{1. ВВЕДЕНИЕ}

В начале 1970-х годов началось бурное развитие многоимпульсных методов в ЯМР твердого тела, которые практически полностью определяют лицо современной радиоспектроскопии [1], [2]. Именно благодаря этим методам ЯМР имеет широчайшее применение: от исследований физики твердого тела и сверхпроводимости до молекулярной биологии и медицины (включая диагностику).

Основным обстоятельством, определившим успех новых методов, явилась возможность фактически неограниченных трансформаций гамильтониана ядерной спиновой системы в соответствии с проектом исследования, что позволило говорить даже и об “алхимии спиновых систем". В то же время сигналы, наблюдаемые при использовании

\footnotetext{
${ }^{*}$ Институт физики им. Л. В. Киренского СО РАН, Красноярск, Россия. E-mail: rsa@iph.krasn.ru, root@iph.krasnoyarsk.su

${ }^{\dagger}$ Институт химической физики им. Н. Н. Семенова РАН, Москва, Россия. E-mail: andylun@orc.ru
} 
импульсных методов ЯМР, определяются различными временнь́ми корреляционными функциями (ВКФ) и, таким образом, подобные исследования имеют сушественное значение для развития статистической механики необратимых процессов: ядерные спиновые подсистемы твердого тела с их точно известным (а часто и заранее спроектированным) гамильтонианом и практически полной изолированностью от остальных степеней свободы образца (решетки) являются, по словам Бломбергена, "великолепной лабораторией статистической физики".

Упомянем здесь лишь о реализованном на практике в ЯМР “обрашении времени" в спиновой системе твердого тела [3], [4], лежашем в основе многих современных экспериментальных методик, и “квантовых вычислениях" (компьютерах), реализуемых с помощью многоимпульсного ЯМР [5].

Одним из наиболее интересных направлений развития ЯМР твердого тела является многоквантовая фурье-спектроскопия [2], [4], [6]-[8]. Все многообразие конкретных реализаций ее методов в конце концов сводится к тому, что после облучения спиновой системы последовательностью радиочастотных импульсов в ней создается несекулярный (по отношению к равновесной намагниченности) гамильтониан, под воздействием которого первоначальная намагниченность перекачивается в различные многоспиновые ВК $\Phi$ (многоспиновые когерентности). Возникшие когерентности метятся с помошњю фазового сдвига пропорционального времени, для чего используется еше один радиочастотный импульс. Возникающий фазовый сдвиг пропорционален $n \varphi$, где $n$ - целое число, а $\varphi-$ фаза, сообшаемая импульсом. Таким образом, в зависимости от $n$ когерентности различают еше и по числу квантов [2]. Затем к системе прикладывается новая импульсная последовательность, изменяюшая знак упомянутого несекулярного гамильтониана, и тем самым реализуется "обрашение времени”, вследствие которого система развивается "вспять". Наблюдение эволюции в зависимости от времени эволюции и фазы $\varphi$ позволяет построить одномерный или двумерный спектр Фурье.

Многоквантовая ЯМР-спектроскопия очень удобна для исследований, например, структуры кластеров или иных локальных структур [9]. В случае же регулярных твердых тел развитие приложений сдерживается, в частности, отсутствием последовательной теории (см., например, [6]-[9]). С другой стороны, временна́я эволюция цепочки многоспиновых многоквантовых ВК $\Phi$ чрезвычайно интересна с точки зрения статистической механики необратимых процессов.

В данной работе указанная задача рассматривается на основе модели с бесконечным радиусом взаимодействия. Предлагаемая модель позволяет получить точное решение поставленной задачи. Полученные результаты согласуются с экспериментальными [4], по крайней мере, качественно.

\section{2. ГАМИЛЬТОНИАН, МОДЕЛЬНЫЙ ГАМИЛЬТОНИАН И ПОСТАНОВКА ЗАДАЧИ}

Физической величиной, непосредственно наблюдаемой в экспериментах по импульсному ЯМР, является намагниченность в системе координат, врашаюшейся с ларморовской частотой $\gamma H_{0}$ вокруг оси $Z$ лабораторной системы, вдоль которой приложено большое постоянное магнитное поле $H_{0}(\gamma$ - гиромагнитное отношение). В простейших экс- 
периментах эта намагниченность, возбуждаемая одним $\pi / 2$-импульсом (т.е. импульсом, длительность $t_{p}$ которого во врашающейся системе координат (BCK) подобрана таким образом, что $\gamma H_{1} t_{p}=\pi / 2$, где $H_{1}$ - амплитуда радиочастотного поля) и называемая сигналом свободной прецессии, пропоршиональна ВКФ:

$$
\Gamma_{0}(t)=\frac{\operatorname{Sp}\left(S_{x}(t) S_{x}\right)}{\operatorname{Sp} S_{x}^{2}}=\frac{\operatorname{Sp}\left(S^{+}(t) S^{-}\right)}{\operatorname{Sp}\left(S^{+} S^{-}\right)},
$$

где $S_{x}=\sum_{i} S_{x_{i}}-x$-компонента суммарного спина системы, удовлетворяющая уравнению Гейзенберга

$$
\begin{gathered}
\dot{S}_{x}=i\left[H_{\mathrm{d}}^{0}, S_{x}\right], \\
S^{+}=S_{x}+i S_{y}, \quad S^{-}=S_{x}-i S_{y} .
\end{gathered}
$$

В уравнении (2) и далее энергии измеряются в частотных единишах, $H_{\mathrm{d}}^{0}$ - секулярная часть диполь-дипольного взаимодействия [10], в основном ответственного за уширение спектров ЯМР в неметаллических диамагнитных кристаллах:

$$
H_{\mathrm{d}}^{0}=\frac{3}{2} \sum_{i \neq j} b_{i j} S_{z_{i}} S_{z_{j}}-\frac{1}{2} \sum_{i \neq j} b_{i j} \vec{S}_{i} \vec{S}_{j}=H_{z z}+H_{\mathrm{ex}}
$$

где

$$
b_{i j}=\frac{\gamma^{2} \hbar}{2 r_{i j}^{3}}\left(1-3 \cos ^{2} \theta_{i j}\right)
$$

$\vec{r}_{i j}$ - вектор, соединяюший спины $i$ и $j, \theta_{i j}$ - угол, образуемый вектором $\vec{r}_{i j}$ с постоянным внешним магнитным полем. Гамильтониан (3) является базовым для "спиновой алхимии", преобразуясь под влиянием радиочастотных импульсов в другие гамильтонианы, представляюшие интерес для исследователя.

Задача вычисления даже и простейшей ВКФ (1) с гамильтонианом (3) чрезвычайно сложна в связи с многочастичностью задачи и отсутствием явного малого параметра в гамильтониане. Сушественньй прогресс был достигнут лишь в последние годы (см., например, работы [11], [12] и ссылки в них). Расчет же ВКФ, определяющих поведение многоспиновых многоквантовых когерентностей, представляется, как будет видно из изложенного, еше более сложной задачей. В связи с этим для упрошения проблемы сделаем основное предположение для исследуемой ниже модели. Пусть коэффициенты $b_{i j}$ не зависят от углов и расстояний, т.е. $b_{i j}=b$ для любых $i, j$. Такого рода подход довольно широко распространен в физике магнитных явлений (см., например [13]-[15]). Теперь гамильтониан (3) перепишется в виде

$$
H_{\mathrm{d}}^{0}=\frac{3}{2} \sum_{i \neq j} b S_{z_{i}} S_{z_{j}}-\frac{1}{2} \sum_{i \neq j} b \vec{S}_{i} \vec{S}_{j}
$$

Сделанное предложение радикально упрошает проблему. Так, при расчете ВKФ (1) для спина $1 / 2$ слагаемое $H_{\text {ex }}$ выпадает из дальнейшего рассмотрения, поскольку слага-

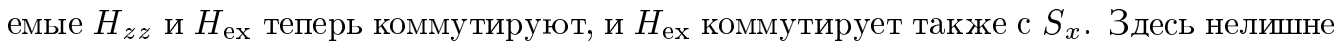


указать, что в работах [6], [7] при расчете коэффициентов, с помощью которых моделировалась диффузия в пространстве Лиувилля, сложность задачи также вынудила авторов прибегнуть к приближению равных взаимодействий. Мы же в настоящей работе (в отличие от [6], [7]) будем рассматривать точную динамику многоспиновых многоквантовых когерентностей в рамках описанной выше модели. Кроме того, следует отметить, что в регулярных твердых телах, в которых каждый спин окружен большим числом эквивалентных соседей, форма ВКФ практически не зависит от величины спина $S$, влияющей на временно́й масштаб [16].

Таким образом, для $S=1 / 2$ гамильтониан (4) при вычислении ВКФ (1) заменяется выражением

$$
H_{\mathrm{d}}^{0}=H=B S_{z}^{2}-\frac{N}{4}, \quad B=\frac{3}{2} b
$$

где $N$ - число спинов в образце. С учетом этого получим

$$
\exp (i H t) S^{+} \exp (-i H t)=\exp \left[i t B\left(S_{z}^{2}-\left(S_{z}-1\right)^{2}\right)\right] S^{+}=\exp \left[2 i t B\left(S_{z}-\frac{1}{2}\right)\right] S^{+}
$$

Слагаемое $-1 / 2$ в операторе эволюции (6) возникает вследствие исключения самого спина из локального поля на нем. Очевидно, что при больших $N$ этим слагаемым можно пренебречь. Кроме того, в этом приближении $S_{z}, S_{x}$ и $S_{y}$ являются числами (а не операторами), каждое из которых распределено по нормальному закону со вторым моментом

$$
\begin{gathered}
\left\langle S_{\alpha}^{2}\right\rangle=\sum_{i} S_{\alpha i}^{2}=\frac{N}{4}, \\
P_{\alpha}\left(S_{\alpha}\right)=\sqrt{\frac{2}{\pi N}} \exp \left(-\frac{2 S_{\alpha}^{2}}{N}\right), \quad \alpha=x, y, z .
\end{gathered}
$$

Вычисление следа в формуле (1) означает усреднение проекций с распределением (8).

Таким образом, легко получим

$$
\Gamma(t)=\exp \left(-\frac{M_{2} t^{2}}{2}\right), \quad M_{2}=\frac{B^{2}}{N} .
$$

При создании и наблюдении многоспиновых и многоквантовых когерентностей в эксперименте, как уже отмечалось, прибегают к следующей последовательности действий: на первом этапе вызывают эволюцию системы с дипольным гамильтонианом (для рассматриваемой модели гамильтонианы (4) или (5)) на временно́м интервале длительностью $t$, затем производят разделение возникших многоспиновых когерентностей по числу (поглошаемых) квантов, одновременно помечая их. Последнее реализуют с помощью поворота на некоторый угол $\varphi$ вокруг оси $x$ BCK. На последнем этапе добиваются эволюции системы с гамильтонианом $-H$ на временно́м интервале длительностью $\tau$. Таким образом, интересуюшая нас ВКФ может быть записана в виде

$$
\Gamma_{\varphi}(t, \tau)=\frac{\operatorname{Sp}\left[U^{+}(\tau) U_{\varphi} U(t) S_{x} U^{+}(t) U_{\varphi}^{+} U(\tau) S_{x}\right]}{\operatorname{Sp} S_{x}^{2}} .
$$


Строго говоря, эксперимент, описанный в работе [4], выполнен с эффективным гамильтонианом, несколько отличающимся от нашего (см. далее формулу (39) и следующее за ней обсуждение). Тем не менее на анализ динамики возникающих многочастичных корреляций эти различия влияют несушественно. На это же указывают и результаты работ [7], [8].

Как будет видно из изложенного ниже, описание эволюции многоквантовых ВКФ (10) оказывается даже для модельного гамильтониана существенно более громоздкой задачей, чем расчет сигнала свободной прецессии (9).

\section{3. РАЗЛОЖЕНИЕ ПО ОРТОГОНАЛЬНЫМ ОПЕРАТОРАМ}

Для того чтобы явно выделить многочастичные когерентности, возникающие в операторе эволюции (6), разложим последний в ряд по ортогональным операторам. В соответствии с методами, предложенными в работах [11], [17], ортогональньй базис будем строить с помощью следующих коммутаторов с гамильтонианом (5):

$$
\begin{gathered}
Q_{1}=\left[H_{\mathrm{d}}^{0}, S_{0}^{+}\right]=2 B \sum_{i} S_{z i} S_{0}^{+}, \\
Q_{2}=\left[H_{\mathrm{d}}^{0},\left[H_{\mathrm{d}}^{0}, S_{0}^{+}\right]\right]=(2 B)^{2} \sum_{j \neq i} S_{z j} S_{z i} S_{0}^{+}+(2 B)^{2} \frac{N}{4} S_{0}^{+} .
\end{gathered}
$$

Индекс 0 у спина фиксирует некоторый выделенньй (любой) спин в решетке. Только первое слагаемое в операторе (12) ортогонально к оператору $S_{0}^{-}$. Совершенно аналогично часть $n$-кратного коммутатора

$$
Q_{n}=\left[H_{\mathrm{d}}^{0},\left[H_{\mathrm{d}}^{0}, \ldots,\left[H_{\mathrm{d}}^{0}, S_{0}^{+}\right] \ldots\right]\right]
$$

ортогональная ко всем предшествуюшим, имеет вид

$$
Q_{n}^{\text {(ort) }}=(2 B)^{n}\left(\frac{N}{4}\right)^{n / 2} O_{n}^{+}
$$

где

$$
O_{n}^{+}=\sum_{i \neq j \neq j \neq \cdots \neq q} S_{z i} S_{z j} \ldots S_{z q} S_{0}^{+}\left(\frac{4}{N}\right)^{n / 2} .
$$

Скалярное произведение вычисляется в соответствии с правилом

$$
\left\langle O_{n}^{+} \mid O_{m}^{-}\right\rangle=\delta_{n m} \operatorname{Sp}\left(S_{0}^{+} S_{0}^{-}\right) n !
$$

Разложим эволюционируюшую поперечную компоненту спина в ряд по набору ортогональных операторов:

$$
U(t) S_{0}^{+} U^{+}(t)=\sum_{n=0} i^{n} A_{n}(t) O_{n}^{+} .
$$


$\mathrm{BK} \Phi A_{n}(t)$ представляют собой многочастичные когерентности. Домножая (16) на $O_{n}^{-}$ и вычисляя скалярное произведение, получим

$$
\begin{aligned}
\operatorname{Sp}\left[S_{0}^{+}(t) O_{n}^{-}\right] & =\operatorname{Sp}\left[\exp \left(2 i B S_{z} t\right) S_{0}^{+} \sum_{i \neq j \neq \cdots \neq 0} S_{z i} S_{z j} \ldots S_{z n} S_{0}^{-}\right]\left(\frac{4}{N}\right)^{n / 2}= \\
& =i^{n} A_{n}(t) n ! \operatorname{Sp}\left(S_{0}^{+} S_{0}^{-}\right)
\end{aligned}
$$

Разложим в ряд экспоненту в формуле (17):

$$
\exp \left(2 i B S_{z} t\right)=\sum_{m} \frac{(2 i B t)^{m}}{m !}\left(\sum_{i} S_{z i}\right)^{m}
$$

и вычислим след. При $m<n$ этот след равен нулю. При $m=n$ мы можем спарить операторы $S_{z j}$ из $O_{n}^{-}$с операторами $S_{z i}$ из разложения экспоненты $n !$ способами. Таким образом, при $m=n$ получим

$$
(2 i B t)^{n} \operatorname{Sp}\left(S_{0}^{+} S_{0}^{-}\right)\left(\frac{n !}{n !}\right)\left(\frac{N}{4}\right)^{n}\left(\frac{4}{N}\right)^{n / 2}
$$

При $m>n$ мы можем выбрать из $\left(S_{z}\right)^{m}$ операторы для спаривания с операторами $S_{z j}$ из $O_{n} m(m-1) \ldots(m-n-1)=m ! /(m-n) !$ способами. Следовательно, для всего ряда получим

$$
\begin{aligned}
\operatorname{Sp}\left(S_{0}^{+} S_{0}^{-}\right)(2 i B t)^{n} & \left\langle\sum_{m \geqslant n} \frac{\left(2 i B t S_{z}\right)^{n-m}}{(m-n) !}\right\rangle\left(\frac{N}{4}\right)^{n}\left(\frac{4}{N}\right)^{n / 2}= \\
& =\operatorname{Sp}\left(S_{0}^{+} S_{0}^{-}\right)(2 i B t)^{n}\left(\frac{N}{4}\right)^{n / 2} \exp \left(-\frac{B^{2} t^{2} N}{2}\right)
\end{aligned}
$$

Подставив выражение (20) в (16), найдем явное выражение для многоспиновой когерентности в исследуемой модели:

$$
A_{n}(t)=(2 B t)^{n}\left(\frac{N}{4}\right)^{n / 2} \exp \left(-\frac{B^{2} t^{2} N}{2}\right)=\left(\sqrt{M_{2}} t\right)^{n} \frac{\exp \left(-M_{2} t^{2} / 2\right)}{n !}
$$

\section{4. ВЫЧИСЛЕНИЕ ИНТЕНСИВНОСТЕЙ МНОГОЧАСТИЧНЫХ КОГЕРЕНТНОСТЕЙ}

Для описания многоспиновых многоквантовых когерентностей представим операторы $S_{x}$, содержащиеся в соотношении (10), через операторы $S^{+}$и $S^{-}$. Тогда

$$
\Gamma_{\varphi}(t, \tau)=\frac{1}{2}\left(\Gamma_{\varphi}^{+-}(t, \tau)+\Gamma_{\varphi}^{++}(t, \tau)+\Gamma_{\varphi}^{-+}(t, \tau)+\Gamma_{\varphi}^{--}(t, \tau)\right)
$$


Рассмотрим первое из слагаемых в правой части (22). Делая циклическую перестановку в следе, получим

$$
\Gamma_{\varphi}^{+-}(t, \tau)=\frac{\operatorname{Sp}\left[U_{\varphi} U(t) S^{+} U^{+}(t) U_{\varphi}^{+} U^{+}(\tau) S^{-} U(\tau)\right]}{\operatorname{Sp}\left(S^{+} S^{-}\right)} .
$$

Подставляя в (23) разложение (16), найдем

$$
\Gamma_{\varphi}^{+-}(t, \tau)=\frac{\operatorname{Sp}\left[U_{\varphi}\left(\sum_{n} i^{n} A_{n}(t)\left(\sum_{0} O_{n}^{+}\right)\right) U_{\varphi}^{+}\left(\sum_{m}(-i)^{m} A_{m}(\tau)\left(\sum_{0^{\prime}} O_{m}^{-}\right)\right)\right]}{\operatorname{Sp}\left(S^{+} S^{-}\right)} .
$$

В соотношении (24) ортогональные операторы просуммированы по всем возможным положениям выделенного спина " 0 ” ( “ $0^{\prime \prime}$ ”), в противном случае функция $\Gamma_{\varphi}^{+-}(t, \tau)$ свелась бы лишь к своей автокорреляционной части, что в рамках рассматриваемой модели было бы некорректно.

Пусть в формуле $(22)$ угол $\varphi=0$. В этом случае слагаемые $\Gamma_{\varphi}^{++}(t, \tau)$ и $\Gamma_{\varphi}^{--}(t, \tau)$ обрашаются в нуль, а у ортогональных операторов в выражении (22) $n=m, 0=0$. Теперь

$$
\Gamma_{\varphi=0}^{+-}(t, \tau)=\sum_{n} A_{n}(t) A_{n}(\tau) i^{n}(-i)^{n} n ! .
$$

Подставляя (21) в (25), найдем

$$
\Gamma_{\varphi=0}^{+-}(t, \tau)=\sum_{n}\left(M_{2} t \tau\right)^{n} \frac{\exp \left[-M_{2}\left(t^{2}+\tau^{2}\right) / 2\right]}{n !}=\exp \left[\frac{-M_{2}(t-\tau)^{2}}{2}\right]
$$

Как и следовало ожидать, при $t=\tau$ получаем $\Gamma_{\varphi=0}^{+-}(\tau, \tau)=1$ - полное "обращение времени". Подействуем теперь на $O_{n}^{+}$из $(11)$ оператором поворота $U_{\varphi}=\exp \left(i \varphi S_{x}\right)$ :

$$
\begin{aligned}
O_{n}^{+}(\varphi)= & \left(\frac{4}{N}\right)^{n / 2} \sum_{\substack{i \neq j \neq \cdots \neq q \neq 0\\
}}\left(S_{z i} \cos \varphi-S_{y i} \sin \varphi\right) \ldots\left(S_{z q} \cos \varphi-S_{y q} \sin \varphi\right) \times \\
& \times\left(S_{0}^{x}+i S_{y 0} \cos \varphi+i S_{z 0} \sin \varphi\right)
\end{aligned}
$$

Последний множитель под знаком суммы в формуле (27) может быть переписан в виде

$$
i S_{z 0} \sin \varphi+\frac{1}{2} S_{0}^{+}(1+\cos \varphi)+\frac{1}{2} S_{0}^{-}(1-\cos \varphi)
$$

Автокорреляционный вклад в (24) получается при совпадении индексов “ 0 ” и " 0 '” и

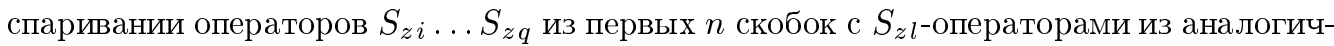
ного набора. Оператор $S_{0}^{+}$спаривается при этом с оператором $S_{0}^{-}$. Тогда получим

$$
\begin{aligned}
\Gamma_{\varphi \text { auto }}^{+-}(t, \tau) & =\sum_{n} \frac{1}{2}\left(M_{2} t \tau\right)^{n} \exp \left[-\frac{M_{2}\left(t^{2}+\tau^{2}\right)}{2}\right](\cos \varphi)^{n} \frac{(1+\cos \varphi)}{n !}= \\
& =\frac{1}{2}(1+\cos \varphi) \exp \left[-\frac{M_{2}\left(t^{2}+\tau^{2}\right)}{2}+M_{2} t \tau \cos \varphi\right]
\end{aligned}
$$


Перекрестный вклад в $(24)$ получится при совпадении индекса " 0 '” у операторов $O_{m}^{-}$ с одним из индексов у операторов $S_{y i}$ в произведении (27) и при совпадении индекса "0"

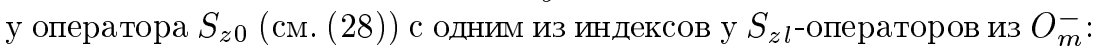

$$
\begin{aligned}
\Gamma_{\varphi \text { cross }}^{+-}(t, \tau) & =-\frac{1}{2} \sum_{n}\left(M_{2} t \tau\right)^{n}(\cos \varphi)^{n-1} \sin ^{2} \varphi \frac{\exp \left[-M_{2}\left(t^{2}+\tau^{2}\right) / 2\right] n}{n !}= \\
& =-\frac{1}{2} \sin ^{2} \varphi\left(M_{2} t \tau\right) \exp \left[M_{2} t \tau \cos \varphi\right] \exp \left[-\frac{M_{2}\left(t^{2}+\tau^{2}\right)}{2}\right] .
\end{aligned}
$$

Складывая (29) и (30), находим

$$
\Gamma_{\varphi}^{+-}(t, \tau)=\frac{1}{2}\left(1+\cos \varphi-M_{2} t \tau \sin ^{2} \varphi\right) \exp \left[-\frac{M_{2}\left(t^{2}+\tau^{2}\right)}{2}+M_{2} t \tau \cos \varphi\right] .
$$

Рассмотрим теперь второе слагаемое из (22):

$$
\Gamma_{\varphi}^{++}(t, \tau)=\frac{\operatorname{Sp}\left[U_{\varphi}\left(\sum_{n} A_{n}(t)\left(\sum_{0} i^{n} O_{n}^{+}\right)\right) U_{\varphi}^{+}\left(\sum_{m} A_{m}(\tau)\left(\sum_{0} i^{m} O_{m}^{+}\right)\right)\right]}{\operatorname{Sp}\left(S^{+} S^{-}\right)} .
$$

При вычислении автокорреляционной части (32) изменения (по сравнению с выгислениями, проведенными при получении соотношения (31)) сводятся к изменению знака перед $\cos \varphi$ вследствие изменения знака перед $i^{m}$ и переходу от второго к последнему слагаемому в формуле (28). Аналогичные изменения произойдут и при изменении перекрестной части. Таким образом, получим

$$
\Gamma_{\varphi}^{++}(t, \tau)=\frac{1}{2}\left(1-\cos \varphi-M_{2} t \tau \sin ^{2} \varphi\right) \exp \left[-\frac{M_{2}\left(t^{2}+\tau^{2}\right)}{2}-M_{2} t \tau \cos \varphi\right]
$$

Два оставшихся в выражении (22) слагаемых являются комплексно-сопряженными к приведенным выражениям (т.е. в данном случае совпадают с ними). Окончательно получим

$$
\begin{aligned}
\Gamma_{\varphi}(t, \tau)= & \frac{1}{2}\left(1+\cos \varphi-M_{2} t \tau \sin ^{2} \varphi\right) \exp \left[-\frac{M_{2}\left(t^{2}+\tau^{2}\right)}{2}+M_{2} t \tau \cos \varphi\right]+ \\
& +\frac{1}{2}\left(1-\cos \varphi-M_{2} t \tau \sin ^{2} \varphi\right) \exp \left[-\frac{M_{2}\left(t^{2}+\tau^{2}\right)}{2}-M_{2} t \tau \cos \varphi\right]
\end{aligned}
$$

Искомая амплитуда $n$-й гармоники, наблюдаемая экспериментально, получается после фурье-преобразования (34):

$$
g_{n}(t, \tau)=(2 \pi)^{-1} \int_{-\pi}^{\pi} d \varphi \exp (\operatorname{in\varphi }) \Gamma_{\varphi}(t, \tau)
$$

Далее можно воспользоваться формулами Эйлера и определением функции Бесселя

$$
I_{n}(x)=\pi^{-1} \int_{0}^{\pi} d t \exp (x \cos t) \cos n t
$$


Изменение знака при $\cos \varphi$ в показателе экспоненты (34) изменяет знак перед функцией Бесселя при нечетном $n$ и не изменяет при четном. Следовательно, при нечетном $n$ два слагаемых в (34) взаимно компенсируются, а при четном складываются. Таким образом, расчеты в рамках избранной модели приводят к появлению когерентностей только четных порядков, как и в экспериментах [4]. Окончательно найдем

$$
\begin{aligned}
g_{2 n}(t, \tau)= & \exp \left[-\frac{M_{2}\left(t^{2}+\tau^{2}\right)}{2}\right]\left[I_{2 n}\left(M_{2} t \tau\right)\left(1-\frac{M_{2} t \tau}{2}\right)+\frac{1}{2} I_{2 n+1}\left(M_{2} t \tau\right)+\right. \\
& \left.+\frac{1}{2} I_{2 n-1}\left(M_{2} t \tau\right)+\frac{1}{4} M_{2} t \tau\left(I_{2 n+2}\left(M_{2} t \tau\right)+I_{2 n-2}\left(M_{2} t \tau\right)\right)\right] .
\end{aligned}
$$

Далее, используя рекуррентные соотношения для функций Бесселя [18], получим

$$
g_{n}(t, \tau)=I_{n}\left(M_{2} t \tau\right)\left(1+\frac{n^{2}}{M_{2} t \tau}\right) \exp \left[-\frac{M_{2}\left(t^{2}+\tau^{2}\right)}{2}\right] .
$$

Отметим, что выражение (38) получено в предположении бесконечно большого числа спинов в системе $N$. В то же время в молекулярных твердых телах очень часто с помощью дейтерирования создают группы изолированных спинов, принадлежащих, как правило, одной молекуле (или ее фрагменту) с высокой симметрией. Межмолекулярный вклад ядерного диполь-дипольного взаимодействия в уширение спектра в таких системах, как правило, практически полностью (или по крайней мере в очень значительной степени) удаляют, а внутримолекулярный вклад адекватно описывается приведенным выше модельным гамильтонианом (4), (5). При этом, однако, необходимо учитывать конечность числа $N$ [19]. Соответствующие формулы приведены в приложении 1.

\section{5. ОБСУЖДЕНИЕ РЕЗУЛЬТАТОВ}

На рис. 1 приведены зависимости $g_{n}(t)$ для гексаметилбензола, построенные с помощью формулы (38). Экспериментально подобные зависимости впервые наблюдались авторами работы [4]. Гамильтониан ядерной спиновой системы, ответственный за ее динамику, имел вид

$$
H_{\mathrm{eff}}=\frac{1}{3}\left(H_{x x}-H_{y y}\right)=\frac{1}{2} \sum_{i \neq j} b_{i j}\left(S_{x i} S_{x j}-S_{y i} S_{y j}\right) .
$$

При этом наблюдавшаяся ВК $\Phi$ была пропорциональна $\operatorname{Sp}\left(S_{z}(t) S_{z}\right)$. Переход от корреляционной функции $z$-компоненты спина к функции $x$-компоненты может быть осуществлен с помощью циклической перестановки осей координат ВСК $x \rightarrow y \rightarrow z$.

Второй момент спектра, задаваемого гамильтонианом (39), связан со вторым моментом спектра поглошения ЯМР соотношением

$$
M_{2 \text { eff }}=\frac{2}{3} M_{2 \text { gMP }}
$$

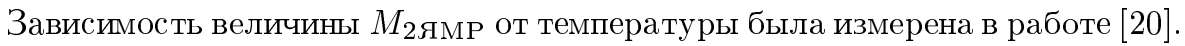


Следует иметь в виду, что гексаметилбензол является молекулярным кристаллом, содержащим бензольные кольца, к которым присоединены метильные группы. Укажем, что четырех-, шести- и более квантовые когерентности не могут образоваться внутри одной метильной группы, и, таким образом, основную роль при их формировании играет диполь-дипольное взаимодействие между различными группами. Именно это относительно слабое взаимодействие и определяет временно́й масштаб для появления корреляций высокого порядка.

При комнатной температуре, отвечаюшей условиям эксперимента, описанного в работе [4], $M_{2 \text { ямР }}=2.5 э^{2}$, что соответствует временно́му масштабу $\approx 33$ мкс. При низких температурах, когда и метильные группы, и бензольные кольца неподвижны, вклад во второй момент ЯМР от протонов собственной метильной группы относится к вкладу от остальных метильных групп как 1 к 6 . При комнатной температуре, когда как метильные групшы, так и бензольные кольца быстро вращаются вокруг осей симметрии, соотношение между указанными выше вкладами неизвестно. При вычислении представленных на рис. 1 зависимостей мы, тем не менее, для оценки предполагали, что соотношение меж ду вкладами во второй момент останется примерно таким же, как и при низких температурах.

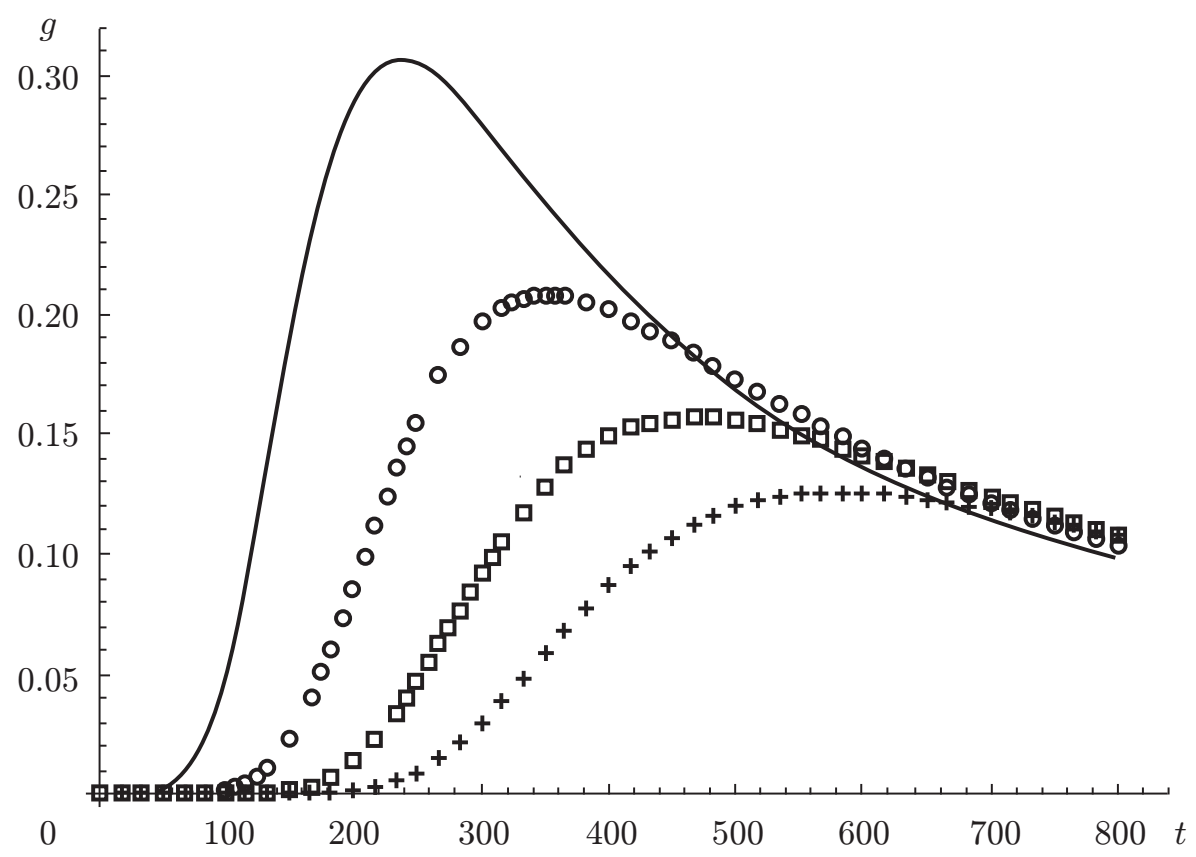

Рис. 1. Удвоенные амплитуды многоспиновых многоквантовых корреляционных функций гексаметилбензола в зависимости от времени; $n=4$ - сплошная кривая, $n=6$ -,$n=8$ $\square, n=10-+$.

Отметим наконец, что эксперименты, описанные в работе [4], выполнялись на поликристаллических образцах, так что для их корректного описания, строго говоря, следовало бы провести усреднение формулы (38) по порошку, что представляется весьма 
затруднительным. В связи с этим мы выбрали второй момент (а с ним и временно́й масштаб), соответствующий поликристаллу, что с учетом приведенных выше замечаний приводит к значению $T^{*} \approx 90$ мкс. Полученные результаты полностью согласуются качественно и неплохо количественно с результатами эксперимента, описанного в работе [4].

В работе [8] для описания результатов эксперимента [4] было получено выражение

$$
g_{n}=\left[I_{n}\left(M_{2} \tau^{2}\right)+\frac{1}{2}\left(I_{n+1}\left(M_{2} \tau^{2}\right)+I_{n-1}\left(M_{2} \tau^{2}\right)\right)\right] \exp \left(-M_{2} \tau^{2}\right)
$$

Усреднение по порошку так же, как и в настоящей работе, не проводилось, а в качестве временно́го масштаба было использовано без каких-либо комментариев значение $T^{*}=57$ мкс, приведшее, однако, к хорошему согласию между теорией и экспериментом. Укажем, что формула (41) была получена для модели, отличной от изложенной вьше. Однако в конце концов разница между формулами (41) и (38) сводится к отсутствию в (41) вклада от перекрестной части (30).

На рис. 2 приведено сравнение результатов расчетов по формулам (38) и (41) с результатами эксперимента [4]. Использованы указанные выше значения временни́х масштабов.

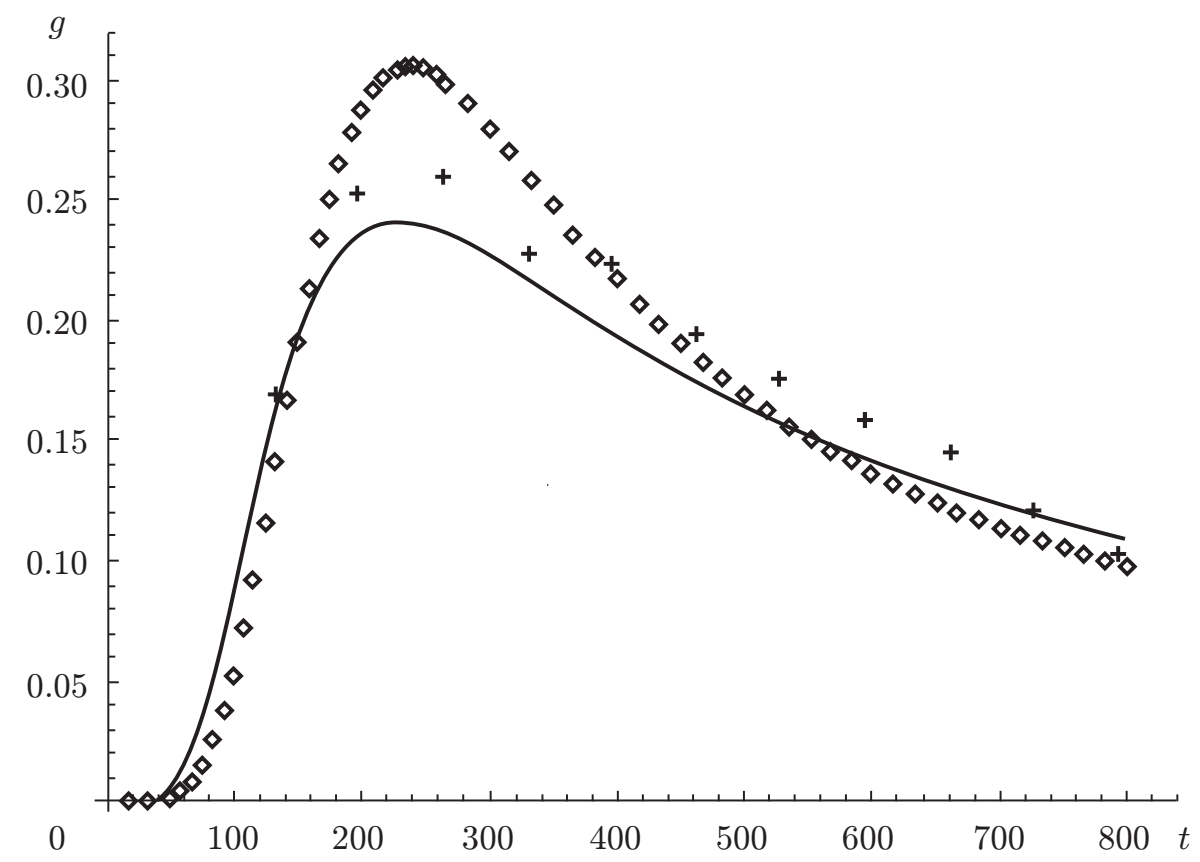

Рис. 2. Удвоенные амплитуды многоспиновых многоквантовых корреляционных функций гексаметилбензола в зависимости от времени для $n=4$. Сплошная кривая - представленная теория, +- расчет по формуле (41), $\square$ - эксперимент [7]. 


\section{6. ЗАКЛЮЧЕНИЕ}

Статистической механикой неравновесных систем давно установлен тот общий факт, что динамическое развитие систем многих взаимодействующих частиц сопровождается образованием многочастичных корреляций (см., например, [21]). Разумеется, ядерные спиновые системы не являются исключением [17]. В то же время возникающие очевидным образом проблемы не позволяют рассчитать сколь-нибудь сложные многочастичные ВКФ для реальных систем. Рассмотренная в настояшей работе решаемая точно модель позволила это сделать. Явно выписан полный набор ортогональных многоспиновых операторов. Найдена зависимость от времени амплитуд многочастичных ВКФ, важных для современной физики магнитного резонанса.

Предложенная нами модель хорошо описывает поведение многочастичных корреляций, по крайней мере, для небольших $n$. В то же время при увеличении числа частищ в корреляционной функции могут появиться расхождения, поскольку у систем с гамильтонианом (39) наблюдается более быстрый рост моментов при увеличении номера (см. приложение 2), чем у функции Гаусса. По-видимому, этим объясняется наблюдавшийся в адамантане [4] очень быстрый рост со временем среднего числа частиц, движение которых коррелированно. Рассмотрение этих эффектов будет проведено дополнительно.

ПРИЛОЖЕНИЕ 1

Получим выражения для интересуюших нас ВКФ, определенных на решетке, содержащей конечное число спинов $N$. Оператор эволюции будет иметь вид

$$
\exp (i H t) S_{0}^{+} \exp (-i H t)=\prod_{j \neq 0}^{N-1}\left(\cos B t+2 i S_{z j} \sin B t\right) S_{0}^{+}
$$

Здесь использованы известные свойства экспоненциальных операторов для спина 1/2. Для ортогональных операторов получим

$$
\begin{gathered}
O_{n}^{+}=\left(\frac{4}{N-1}\right)^{n / 2} \sum_{i \neq j \neq \cdots \neq q \neq 0} \overbrace{S_{z i} S_{z j} \ldots S_{z q}}^{n} S_{0}^{+}, \quad n<N, \\
\operatorname{Sp}\left(O_{n}^{+} O_{m}^{-}\right)=\frac{1}{(N-1)^{n}} \delta_{n m} \frac{n !(N-1) !}{(N-1-n) !} \operatorname{Sp}\left(S_{0}^{+} S_{0}^{-}\right) .
\end{gathered}
$$

Разлагая оператор эволюции в ряд по ортогональным операторам, найдем

$$
\exp (i H t) S_{0}^{+} \exp (-i H t)=i^{n} \sum_{n=0}^{N-1} A_{n}(t) O_{n}^{+}
$$

где

$$
A_{n}(t)=\frac{1}{n !}(N-1)^{n / 2}(\sin B t)^{n}(\cos B t)^{N-n-1} .
$$


Таким образом, искомая ВКФ представляется в виде

$$
\Gamma_{\varphi}^{+-}(t, \tau)=\frac{\operatorname{Sp}\left[U_{\varphi}\left(\sum_{n=0}^{N-1}(i)^{n} A_{n}(t) \sum_{0} O_{n}^{+}\right) U_{\varphi}^{+}\left(\sum_{m=0}^{N-1}(-i)^{m} A_{m}(\tau) \sum_{0^{\prime}} O_{m}^{+}\right)\right]}{\operatorname{Sp}\left(S^{+} S^{-}\right)}
$$

Для автокорреляционной части получим

$$
\begin{aligned}
\Gamma_{\varphi \text { auto }}^{+-}(t, \tau) & =(1+\cos \varphi) \sum_{n} \frac{1}{2(N-1)^{n}} \frac{n !(N-1) !}{(N-1-n) !} A_{n}(t) A_{n}(\tau) \cos ^{n} \varphi= \\
& =\frac{1}{2}(1+\cos \varphi)(\cos B t \cos B \tau+\cos \varphi \sin B t \sin B \tau)^{N-1},
\end{aligned}
$$

а для перекрестной части

$$
\begin{aligned}
\Gamma_{\varphi \operatorname{cross}}^{+-}(t, \tau)= & -\frac{1}{2} \sin ^{2} \varphi \sum_{n}^{N-1} \frac{(N-1) !}{(N-n-1) !(n-1) !} \cos ^{n-1} \varphi(\cos B t \cos B \tau)^{N-n-1} \times \\
& \times(\sin B t \sin B \tau)^{n}=-\frac{1}{2}(N-1) \sin ^{2} \varphi \sin B t \sin B \tau \times \\
& \times(\cos B t \cos B \tau+\cos \varphi \sin B t \sin B \tau)^{N-2} .
\end{aligned}
$$

Как и прежде, выражение для $\Gamma_{\varphi}^{++}(t, \tau)$ получается с помощью изменения знака перед $\cos \varphi$, вследствие чего сумма будет содержать только четные степени $\cos \varphi$. Окончательно получаем

$$
\begin{aligned}
\Gamma_{\varphi}(t, \tau)= & \frac{1}{2}(1+\cos \varphi)(\cos B t \cos B \tau+\cos \varphi \sin B t \sin B \tau)^{N-1}- \\
& -\frac{1}{2}(N-1) \sin B t \sin B \tau(\cos B t \cos B \tau+\cos \varphi \sin B t \sin B \tau)^{N-2}+ \\
& +\frac{1}{2}(1-\cos \varphi)(\cos B t \cos B \tau-\cos \varphi \sin B t \sin B \tau)^{N-1}- \\
& -\frac{1}{2}(N-1) \sin B t \sin B \tau(\cos B t \cos B \tau-\cos \varphi \sin B t \sin B \tau)^{N-2}
\end{aligned}
$$

ПРИЛОЖКЕНИЕ 2

В модели с бесконечным радиусом гамильтониан (39) после циклической замены переменных $S_{x}, S_{y}$ и $S_{z}$ можно записать в виде

$$
H=\frac{1}{2} b\left(S_{z}^{2}-S_{y}^{2}\right)
$$

В дальнейшем будем полагать $b=1$, чему соответствует очевидный выбор масштаба временно́й шкалы. Уравнениям движения

$$
\dot{S}_{x}(t)=-2 S_{z} S_{y}, \quad \dot{S}_{y}(t)=S_{z} S_{x}, \quad \dot{S}_{z}(t)=S_{y} S_{x}
$$


соответствуют два интеграла движения:

$$
S_{x}^{2}+S_{y}^{2}+S_{z}^{2}=S^{2}, \quad \frac{1}{2}\left(S_{z}^{2}-S_{y}^{2}\right)=E .
$$

Аналогичные уравнения получены в работе [14] для системы классических магнитных моментов. Объяснение причин совпадения динамических свойств систем, состоящих из квантовых и классических магнитных моментов, в пределе бесконечного числа ближайших соседей было дано ранее в работе [16]. Следовательно, соответствующие результаты работы [14] могут быть распространены на нашу спиновую систему.

Решением системы уравнений (2.1) являются [14] эллиптические функции Якоби:

$$
\begin{aligned}
& S_{x}(t)=a_{x} \operatorname{sn}\left(\Omega t+\varphi_{0} k\right), \\
& S_{y}(t)=a_{y} \operatorname{dn}\left(\Omega t+\varphi_{0} k\right), \\
& S_{z}(t)=a_{z} \operatorname{cn}\left(\Omega t+\varphi_{0} k\right),
\end{aligned}
$$

где

$$
\begin{gathered}
a_{x}^{2}=S^{2}-2 \varepsilon, \quad a_{y}^{2}=\frac{1}{2}\left(S^{2}+2 \varepsilon\right), \quad a_{x}^{2}=\frac{1}{2}\left(S^{2}-2 \varepsilon\right), \\
\Omega^{2}=2 \varepsilon+S^{2}, \quad K^{2}=\frac{S^{2}-2 \varepsilon}{2 \varepsilon+S^{2}}, \quad \varepsilon=|E| .
\end{gathered}
$$

Эти параметры вместе с фазой $\varphi_{0}$ определяются значениями спиновых проекций при $t=0$. Полученные траектории $S_{x}(t), S_{y}(t), S_{z}(t)$ из системы (2.3) следует усреднить по всем возможным начальным значениям, т.е. по распределениям с гауссовскими функциями плотности.

Решение системы уравнений (2.1) можно искать и в виде временни́х рядов:

$$
S_{x}(t)=\sum_{n=0}^{\infty} X_{n} t^{n}, \quad S_{y}(t)=\sum_{n=0}^{\infty} Y_{n} t^{n}, \quad S_{z}(t)=\sum_{n=0}^{\infty} Z_{n} t^{n} .
$$

Подставив (2.4) в (2.1) и приравняв коэффициенты при равных степенях времени, получаем систему рекуррентных уравнений

$$
\begin{aligned}
(n+1) X_{n+1} & =-2 \sum_{m=0}^{n} Y_{n-m} Z_{m} \\
(n+1) Y_{n+1} & =\sum_{m=0}^{n} Z_{n-m} X_{m} \\
(n+1) Z_{n+1} & =\sum_{m=0}^{n} X_{n-m} Y_{m}
\end{aligned}
$$

с начальными условиями $X_{0}=S_{x}(0), Y_{0}=S_{y}(0), Z_{0}=S_{z}(0)$. Тогда коэффициенты $X_{n}$ получаются в виде суммы

$$
X_{n}=\sum_{m, q, k}^{m+q+k=n+1} C_{m q k}^{n} S_{x}^{m} S_{y}^{q} S_{z}^{k}
$$


Аналогичные выражения получаются и для $Y_{n}, Z_{n}$.

Приведем отличные от нуля коэффициенты $C_{m q k}{ }^{(n)}$ для четырех первых порядков:

$$
\begin{gathered}
C_{11}^{(1)}=-2, \quad C_{102}^{(2)}=C_{120}^{(2)}=-1, \quad C_{013}^{(3)}=C_{031}^{(3)}=\frac{4}{3 !}, \quad C_{211}^{(3)}=-\frac{8}{3 !}, \\
C_{122}^{(4)}=7 \cdot \frac{8}{4 !}, \quad C_{104}^{(4)}=C_{140}^{(4)}=\frac{4}{4 !}, \quad C_{302}^{(4)}=C_{320}^{(4)}=-\frac{8}{4 !} .
\end{gathered}
$$

Соотношение (6) позволяет найти моменты корреляционной функции:

$$
M_{2 n}=\left.(-1)^{n}\left\langle S_{x}^{2}\right\rangle^{-1} \frac{d^{2 n}\left\langle S_{x}(t) S_{x}\right\rangle}{d t^{2 n}}\right|_{t=0}=(-1)^{n}\left\langle\left.\frac{d^{2 n} S_{x}(t)}{d t^{2 n}}\right|_{t=0} S_{x}\right\rangle\left\langle S_{x}^{2}\right\rangle^{-1}
$$

Обозначение $\langle\ldots\rangle$, как и в формуле (7), есть усреднение по начальным условиям с гауссовской функцией распределения. Поскольку

$$
\left.\frac{d^{n} S_{x}(t)}{d t^{n}}\right|_{t=0}=X_{n} n !
$$

подставляя (2.9) в (2.8) и усредняя по независимым гауссовским случайным переменным $S_{x}, S_{y}$ и $S_{z}$, находим

$$
M_{2}=4\left\langle S_{x}^{2}\right\rangle, \quad M_{4}=2\left(M_{2}\right)^{2}, \quad M_{6}=9\left(M_{2}\right)^{3}, \quad M_{8}=117\left(M_{2}\right)^{4} .
$$

Отметим, что для гауссовского распределения $\varphi(x)=\left(1 / M_{2}\left(2 \pi^{1 / 2}\right)\right) \exp \left(-0.5 x^{2} / M_{2}^{2}\right)$ моменты подчиняются соотношению $\mu_{2 k}=1 \cdot 3 \cdot \ldots \cdot(2 k-1) M_{2}^{2 k}$, где $k=1,2, \ldots$

При помощи (2.6) можно определить не только моменты, но и ортогональные операторы в полной аналогии с тем, как это было сделано в основном тексте. Для этого следует подставить в (2.6) $S_{\alpha}=\sum_{i} S_{\alpha i}, \alpha=x, y, z$, и сохранить в суммах только операторы, отвечающие спинам, находящимся в разных узлах.

Благодарности. Работа выполнена при финансовой поддержке РФФИ, грант № 02-02-17463. 


\section{Список литературы}

[1] У. Хеберлен, М. Меринг. ЯМР высокого разрешения в твердых телах. М.: Мир, 1980.

[2] Р. Эрнст, Джс. Боденхаузен, А. Вокаун. ЯМР в одном и двух измерениях. М: Мир, 1990.

[3] R. H. Schneider, H. Schmiedel. Phys. Lett. A. 1969. V. 30. P. 298; W. K. Rhim, A. Pines, J.S. Waugh. Phys. Rev. B. 1971. V. 3. P. 684.

[4] J. Baum, M. Munovitz, A. N. Garroway, A. Pines. J. Chem. Phys. 1985. V. 83. P. 2015.

[5] K. А. Валиев, А. А. Кокин. Квантовые компьютеры: надежды и реальность. Ижевск: НИЦ "Регулярная и хаотическая динамика", 2001.

[6] M. Munovitz, A. Pines, M. Mehring. J. Chem. Phys. 1987. V. 86. P. 3172.

[7] M. Munovitz, M. Mehring. Chem. Phys. 1987. V. 116. P. 79.

[8] А.К. Хитрин. ЖЭТФ. 1996. Т. 109. С. 2173.

[9] P. K. Wang, J. P. Ansermet, Ch. P. Slichter et al. Science. 1986. V. 34. P. 35.

[10] А. Абрагам. Ядерный магнетизм. М.: ИЛ, 1963.

[11] В. Л. Боднева, А.А. Лундин, А.А. Милютин. ТМФ. 1996. Т. 106. С. 452; J. Jensen. Phys. Rev. B. 1995. V. 52. P. 9611.

[12] В. Е. Зобов, А. А. Лундин, О.Е. Родионова. ЖЭТФ. 2001. Т. 120. С. 619; В. Е. Зобов, M. A. Попов. ТМФ. 2003. Т. 136. С. 463.

[13] R. Dekeyser, M. H. Lee. Phys. Rev. B. 1979. V. 19. P. 265.

[14] J. M. Liu, G. Muller. Phys. Rev. A. 1990. V. 42. P. 5854.

[15] R. Dekeyser, M. H. Lee. Phys. Rev. B. 1991. V. 43. P. 8123; 1991. V. 43. P. 8131.

[16] A. A. Lundin, V. E. Zobov. J. Magn. Res. 1977. V. 26. P. 229.

[17] F. Lado, J. D. Memory, G. W. Parker. Phys. Rev. B. 1971. V. 4. P. 1406.

[18] М. А. Абрамовиц, И. А. Стиган. Справочник по специальным функциям с формулами, графиками и математическими таблицами. М.: Наука, 1979.

[19] M. Munovitz, A. Pines. Adv. Chem. Phys. 1987. V. 6. P. 1.

[20] E. R. Andrew. J. Chem. Phys. 1950. V. 18. P. 607.

[21] Р. Балеску. Равновесная и неравновесная статистическая механика. Т. 2. М.: Мир, 1978.

Поступила в редакцию 8.XII.2003 г. 